\title{
Observational Evidence of the Association Between Handgrip Strength, Hand Dexterity, and Cognitive Performance in Community-Dwelling Older Adults: A Systematic Review
}

Kimi Estela Kobayashi-Cuya ${ }^{1,2}$, Ryota Sakurai ${ }^{1}$, Hiroyuki Suzuki ${ }^{1}$, Susumu Ogawa ${ }^{1}$, Toru Takebayashi ${ }^{2}$, and Yoshinori Fujiwara ${ }^{1}$

${ }^{1}$ Research Team for Social Participation and Community Health, Tokyo Metropolitan Institute of Gerontology, Tokyo, Japan

${ }^{2}$ Department of Preventive Medicine and Public Health, School of Medicine, Keio University, Tokyo, Japan

Received March 1, 2017; accepted August 8, 2017; released online March 10, 2018

\begin{abstract}
Background: Deterioration of hand motor function is a possible risk factor of cognitive impairment in older adults. Despite a growing body of research, a lack of clarity exists regarding the relationships. This review offers a synthesis of existing observational studies evaluating the associations of handgrip strength and hand dexterity with cognitive performance in community-dwelling older adults.

Methods: PubMed, PsycINFO, and ScienceDirect were systematically searched (search dates: 1990-2016), and relevant articles were cross-checked for related and relevant publications.

Results: Twenty-two observational studies assessed the association of handgrip strength or hand dexterity with cognitive performance; none evaluated handgrip strength and hand dexterity together. Handgrip strength was associated with global cognition, mostly assessed using the Mini-Mental State Examination, cross-sectionally and longitudinally. Also, one crosssectional and three longitudinal studies found an association with cognitive domains, such as language, memory, visuospatial ability, working memory, and processing speed. Hand dexterity was only assessed cross-sectionally in four studies. These studies found an association with cognitive domains, such as executive function.

Conclusions: Although handgrip strength was associated with cognitive performance, it is unclear which variable at baseline affects the other in the long-term. Cross-sectional studies indicate an association between hand dexterity and cognitive performance, yet longitudinal studies are needed to elucidate this association. The interaction effects of both decreased grip strength and hand dexterity on cognitive performance is still unclear; therefore, future studies will need to consider the interaction of the three variables cross-sectionally and longitudinally.
\end{abstract}

Key words: hand motor function; handgrip strength; hand dexterity; cognitive function; community-dwelling older adults

Copyright @ 2018 Kimi Estela Kobayashi-Cuya et al. This is an open access article distributed under the terms of Creative Commons Attribution License, which permits unrestricted use, distribution, and reproduction in any medium, provided the original author and source are credited.

\section{INTRODUCTION}

Cognitive impairment in late adulthood is one of the causes leading to loss of independence ${ }^{1,2}$ and dementia, ${ }^{3,4}$ which in turn represents an economic burden to the national public health and social welfare. ${ }^{5}$ Several studies in public health and epidemiology have aimed to understand the causes leading to this condition in the older population, ${ }^{6,7}$ its early detection, ${ }^{8}$ and how to prevent it from worsening. ${ }^{9}$ Cognitive decline has been related to the aging process ${ }^{10-12}$; however, there is still a thin line between what is considered normal and pathological cognitive aging. ${ }^{13,14}$ Identification of measurable indicators associated with cognitive impairment in healthy older adults would contribute to the early detection - and possibly prevention - of pathological cognitive decline, such as mild cognitive impairment (MCI), and would foster intervention programs aiming to maintain cognitive processes related to independent living in older adults.
Behavioral research ${ }^{15,16}$ has recently paid much attention to impairment of gait function as a potential motor risk factor for impairment in cognitive function; however, decreased hand motor function, similar to gait function, is also a possible candidate risk factor of cognitive impairment because of its association with cortical brain activity. ${ }^{17-22}$ This association may rely on common neural processes shared between the cognitive and motor areas of the central nervous system and the motor neurons of the peripheral nervous system ${ }^{23,24}$; in other words, it can be hypothesized that the neural circuitry of the hand motor function may associate with that of cognitive performance. This is well supported by the findings that Alzheimer's disease (AD) patients performed hand motor tasks worse than MCI patients who, in turn, performed worse than older adults without cognitive impairment. ${ }^{10,25,26}$ However, the association between hand motor function and cognition is still unclear, as the hand is not only limited to activities where strength is needed, but it is also 
relevant for performing fine and complex activities that require different skills to perform a specific action, such as precision, ${ }^{27}$ speed, ${ }^{28}$ aiming, ${ }^{29}$ and tracing. ${ }^{30}$ Therefore, handgrip strength and hand dexterity, representing hand motor function in this review, seem to be crucial components of functional independence and cognitive maintenance in older adults. This study offers a narrative synthesis of existing observational studies evaluating the association of hand motor function, including handgrip strength and hand dexterity, with cognitive performance in community-dwelling healthy older adults.

\section{METHODS}

\section{Search strategy}

Database searches of PubMed, PsycINFO, and ScienceDirect were conducted using the same search strategy consisting of two combined keyword formulas:

Formula 1 (F1): (elderly OR older adults) AND (handgrip strength OR grip strength OR grasp strength OR grasping power OR grip force) AND (cognitive function)

Formula 2 (F2): (elderly OR older adults) AND (hand dexterity OR dexterity OR hand ability OR manual ability OR motor skill) AND (cognitive function)

A third formula (F3) combining F1 and F2 was considered in a preliminary search conducted in PubMed as the purpose of this review is to examine previous studies evaluating the association between handgrip strength, hand dexterity and cognitive performance. However, only two formulas (F1 and F2) are reported in this review because all findings in F3 were duplicated in F2. The keywords used for handgrip strength were handgrip strength, grip strength, grasp strength, grasping power, and grip force; the keywords used for hand dexterity were hand dexterity, dexterity, hand ability, manual ability, and motor skill. The searches were performed in December 2016, and the selection of the articles were limited to those published in English between January 1990 and December 2016. A grey literature search was performed using Google and Google Scholar. Hand searching through citations and references of relevant articles was also undertaken. The limiters used were year of publication (1990-2016), language (English), population (humans), journal articles (peer reviewed), observational studies (quantitative, qualitative, longitudinal, follow-up, prospective and retrospective), and age (middle aged to $\geq 80$ years old).

\section{Inclusion and exclusion criteria \\ Population}

Studies considering middle-aged subjects and over were included in the review (middle-aged: 45-64 years, aged: $\geq 65$ years, $\geq 80$ years), excluding post-mortem studies. This study reviewed previous observational studies examining the relationship between hand motor function and age-related cognitive impairment, not pathological deficits, among community-dwelling older adults; therefore, studies including patients with neurological disorders were excluded.

\section{Study design}

This review investigated the observational evidence of the association between age-related changes in fine upper motor performance and cognitive impairment; therefore, intervention studies were not included in the analysis. Observational studies, including cross-sectional studies and longitudinal studies, were included. Case studies-where a cognitive control group was included and whose data was separately analyzed-were also included in this review.

\section{Quality assessment}

Titles and abstracts were independently searched for relevance (KK). The full-text publications of the selected studies were reviewed against the inclusion criteria, and the reasons for exclusion at this stage were recorded (Figure 1). Several meetings with the team members (KK, RS, YF, HS, and SO) were held to discuss, verify, and agree on the selection of the articles.

\section{Data analysis}

An evidence table was constructed to help organize and summarize the information of the studies included in the review. The information extracted was setting, number of participants, anthropometric data, cognitive and hand function measurements. A narrative synthesis was completed due to the different measures of cognition and hand function tests used in the selected studies, which made the body of evidence unsuitable for meta-analysis.

\section{RESULTS}

The search results and reasons for exclusion are presented in the PRISMA flow diagram in Figure 1. After applying the inclusion and exclusion criteria, 62 articles were identified from PubMed, PsycINFO, and ScienceDirect databases. No studies evaluated the interaction between the three variables in question. Instead, 22 articles reported the association in pairs; that is, they reported the associations between handgrip strength and cognitive performance or hand dexterity and cognitive performance (Table 1 and Table 2).

Of the 22 articles included in the review, 11 were crosssectional studies (Table 1) and 11 were longitudinal studies (Table 2). Concerning the cross-sectional studies, seven studies included handgrip strength and four studies included hand dexterity, whereas all longitudinal studies included only handgrip strength in their analyses.

Hand dexterity was measured with peg tests (Table 1) and handgrip strength was measured with hand dynamometers, as part of functional health tests and as a composite measure of frailty in some studies (Table 1 and Table 2). MMSE was used as a measurement of global cognitive function and to describe the cohort in some studies. ${ }^{31-33}$ The cognitive tests used in these studies were categorized in this review by cognitive domains (Table 3) and were commonly assessed using the MMSE.

\section{Cross-sectional studies}

\section{Handgrip strength and cognitive function}

Six studies ${ }^{32-37}$ reported significant cross-sectional associations between cognitive performance and handgrip strength in community-dwelling older adults (Table 1), whereas one study ${ }^{31}$ did not find any association. The studies reported on 7,335 healthy community-dwelling older adults with ages ranging from 49 to 100 years. The proportion of males was about $44.6 \%$, excluding the study that included only women. ${ }^{36}$ Years of education varied greatly among studies, ranging from elementary school not completed to 1 year and over of undergraduate studies. The studies used different kinds of handgrip strength devices and different approaches to analyze the association between handgrip strength and cognitive performance.

A study using the MMSE and Animal Naming tests ${ }^{34}$ divided the cognitive data into tertiles according to their scores in each 


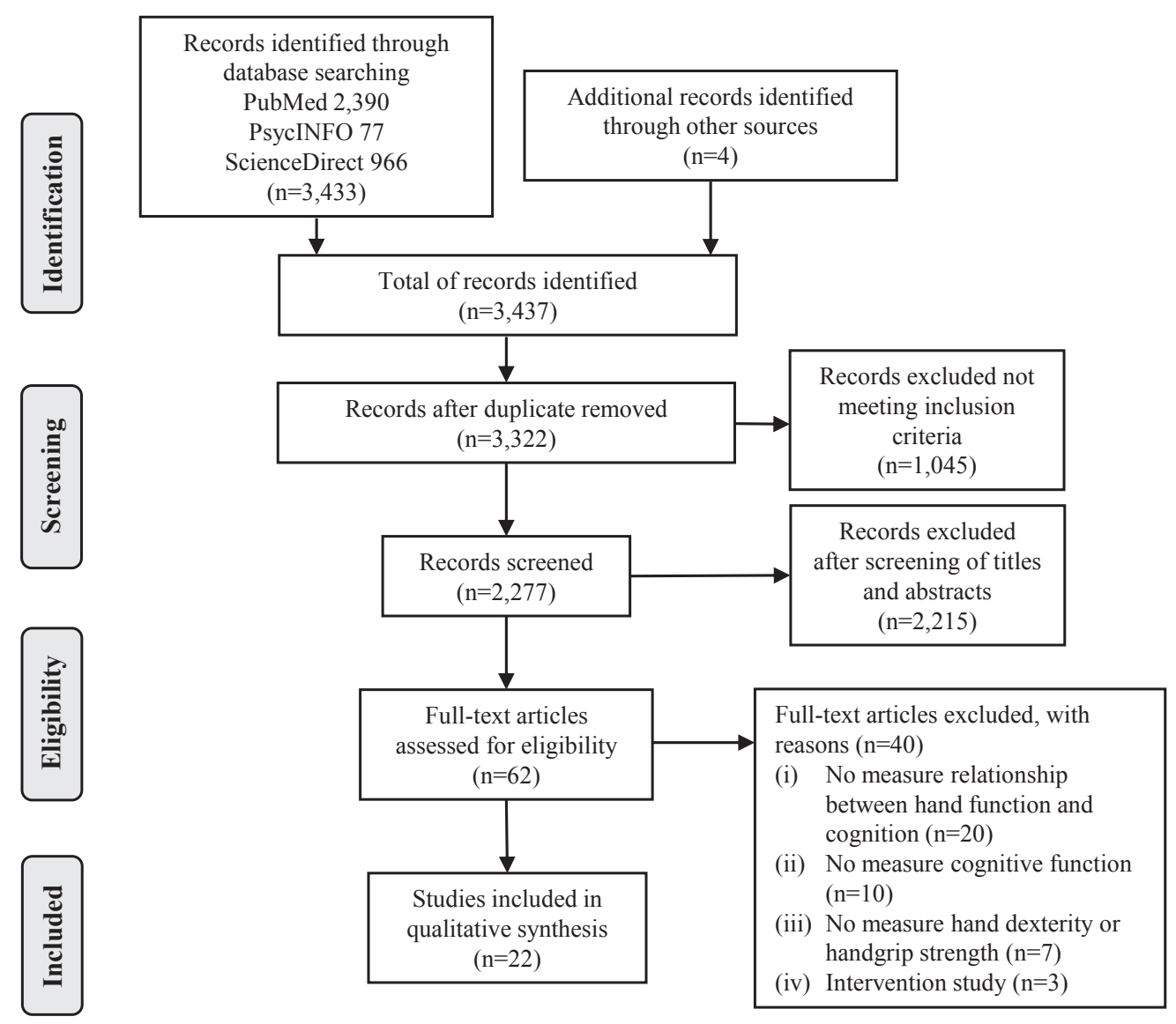

Figure 1. PRISMA flow diagram for the systematic review

test. The study found that handgrip strength was significantly different among the three tertiles of both cognitive tests; that is, the lowest tertile of the cognitive performance in both tests was associated with the lowest grip strength. Another study divided the handgrip strength data into tertiles and found a significant correlation between lower handgrip strength and lower MMSE scores. ${ }^{37}$ Handgrip strength was also correlated with successful aging (SA), defined as an MMSE score of $\geq 24$, along with two other measurements. ${ }^{33}$ Other studies ${ }^{31,32,35,36}$ subdivided their data into cognitively normal and impaired using the following cut-off scores: one study ${ }^{35}$ used the Chinese version of the Community Screening Instrument of Dementia (CSI-D) with a cut-off of 28.4, and the other three studies ${ }^{31,32,36}$ used the MMSE with a cut-off of less than 24 to determine cognitive impairment.

The studies using the full scores of the cognitive tests ${ }^{32,35,36}$ found that handgrip strength performance was significantly lower in the cognitively impaired subjects, whereas the study using a subtest of the MMSE, ${ }^{31}$ the 3-Word Delayed Recall, to differentiate cognitively impaired subjects found no significant association between cognitive performance and handgrip strength. Hand dexterity and cognitive function

The four cross-sectional studies in this category ${ }^{28,38-40}$ reported an association between hand dexterity and cognitive performance (Table 1). The studies reported on 370 healthy communitydwelling older adults, with ages varying from 23 to 93 years. The proportion of males ranged from $28.6 \%$ to $70 \%$, and years of education ranged from 8 to 17 years.

To assess hand dexterity, Yozbatiran et $\mathrm{al}^{38}$ utilized the ninehole peg test (9-HPT), a short-time measurement of upper extremity function. ${ }^{41}$ This study found a significant association between hand dexterity and the Paced Auditory Serial Addition Test (PASAT), a cognitive test used to assess attention, processing speed, and working memory. ${ }^{42,43}$ The Grooved Pegboard Test (GPT), a short neuropsychological test, was used by two studies ${ }^{39,40}$ to assess hand dexterity. In these studies, GPT was analyzed as the time in seconds to complete the test, and the data was divided into right and left hands, with one study ${ }^{40}$ including a composite score (combined scores of right and left hands). In another hand dexterity study, ${ }^{28}$ dexterity was examined with a modified version of the Purdue Pegboard Test (PPT). Hand dexterity was analyzed by assessing the time, angular displacement, and angular velocity of the right hand when reaching, grasping, transporting, and inserting pins and washers.

The data shown in the present review includes only that of healthy older adults; however, it is important to mention that two of these studies ${ }^{38,40}$ included neuro-muscular disease patients in their measurements and found a significant association between hand dexterity and cognitive performance. The healthy older adults were the control groups in both of these studies.

\section{Longitudinal studies}

Most of the longitudinal studies examining the association between handgrip strength and cognitive performance indicated a significant association (Table 2) ${ }^{44-53}$ The studies reported on 16,531 community-dwelling older adults, with ages ranging from 46 to 100 years. The proportion of males was about $57.8 \%$, excluding the study that included only women. ${ }^{52}$ Years of 


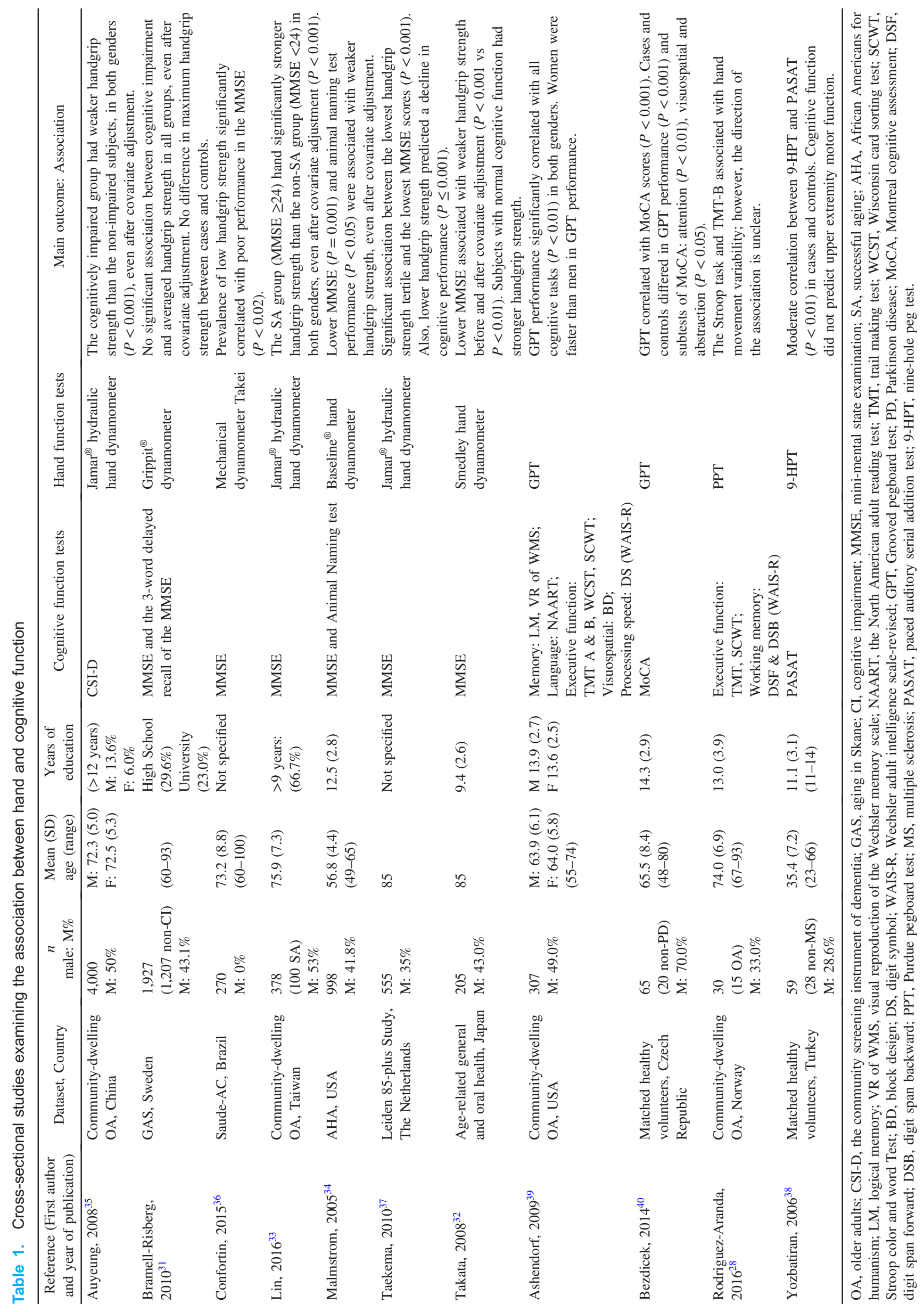




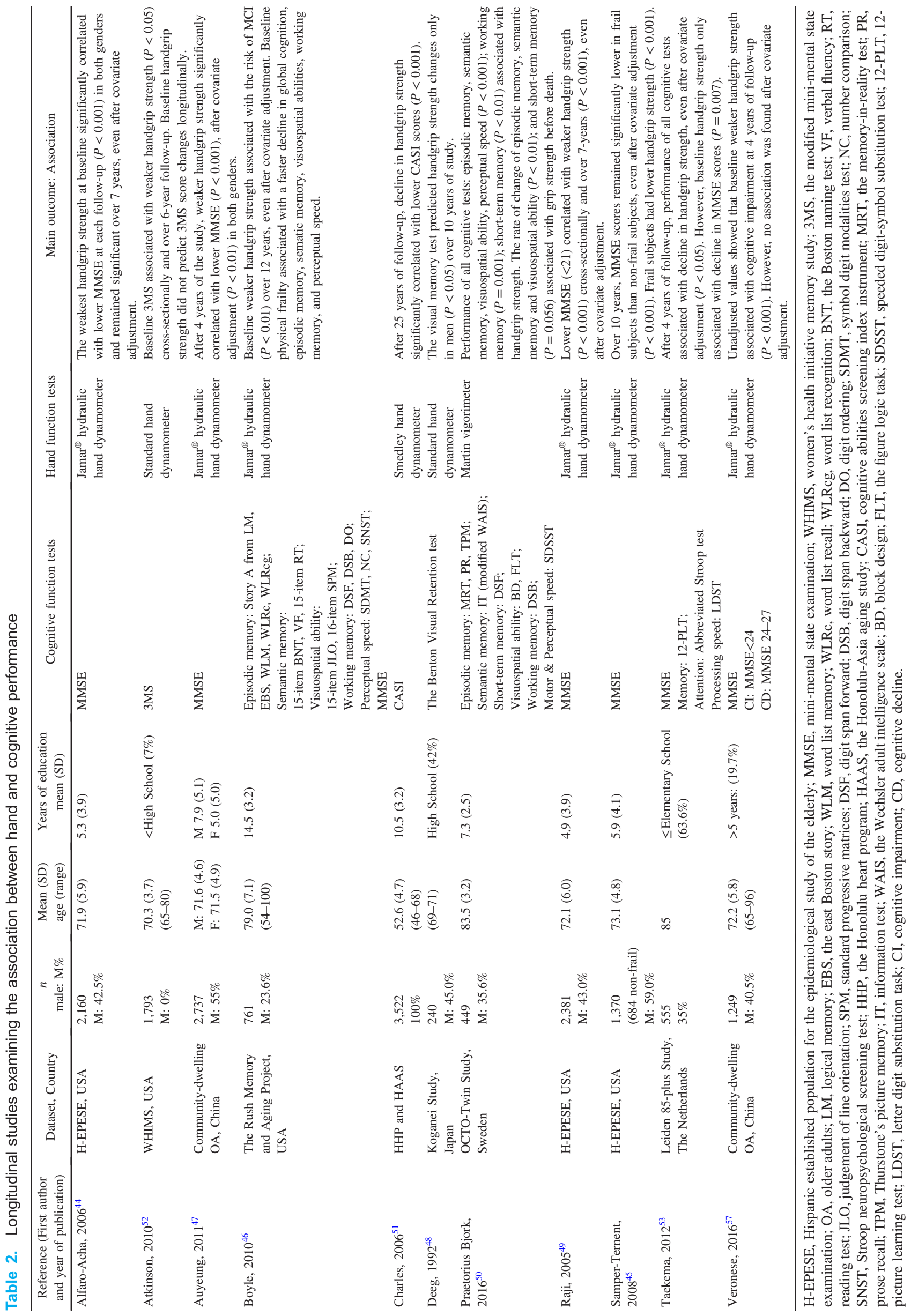


education greatly varied among studies, ranging from elementary school not completed to 1 year and over of undergraduate studies.

Although the association between these two variables has been demonstrated, it is unclear which variable at baseline influences the other in the long-term. Some of the studies ${ }^{48-50,52,53}$ indicated that having a lower cognitive performance at baseline influences the changes in handgrip strength in the long-term. More specifically, Deeg et $\mathrm{al}^{48}$ reported that scores in the Benton Visual Retention test, ${ }^{54}$ a short-term memory test, ${ }^{55}$ predicted changes in handgrip strength over 10 years in Japanese older men, whereas no significant association was seen in women. Another study ${ }^{49}$ indicated that subjects with low baseline MMSE scores, who tended to be older, less educated, and more depressed, had a significantly greater decline in handgrip strength over 7 years than their counterparts, even after covariate adjustment, including age, sex, education, and medical conditions. In addition, a study evaluating various cognitive domains at baseline $\mathrm{e}^{50}$ reported a significant association with handgrip strength decline in older adults aged 80 years and over. A similar finding was suggested in a study evaluating the association of baseline cognitive domains, ${ }^{53}$ such as global cognition, memory, attention, and processing speed, with handgrip strength. Finally, a study using the modified MMSE (3MS) ${ }^{52}$ found that low $3 \mathrm{MS}$ scores at baseline significantly predicted decline in handgrip strength in older women over time; on the other hand, having a lower handgrip strength at baseline did not predict any longitudinal changes in cognitive scores.

In contrast, other studies ${ }^{44-47,51,53}$ suggested that subjects with the lowest handgrip strength at baseline had significantly more cognitive decline and risk of MCI in the long-term than those with higher handgrip strength. In a 25 -year follow-up study, ${ }^{51}$ handgrip strength was significantly associated with the Cognitive Abilities Screening Index Instrument (CASI), a cognitive test that measures various cognitive domains and is considered more sensitive to variations in cognitive impairment than the MMSE. ${ }^{56}$ On the other hand, other studies ${ }^{44-47,53}$ used the MMSE test to measure cognitive performance, with two of them ${ }^{46,53}$ also including different kinds of cognitive tests. Although the studies suggested an association between baseline handgrip strength and changes in $\mathrm{MMSE}^{44,45,47}$ and other cognitive variables, ${ }^{46}$ Taekema et $\mathrm{al}^{53}$ only found an association with MMSE changes and not with other cognitive variables, such as memory, attention, and processing speed.

Contrary to the findings discussed above, Veronese et $\mathrm{al}^{57} \mathrm{did}$ not find a significant association between baseline handgrip strength and the onset of cognitive impairment. It is important to note that, while unadjusted values indicate a significant association $(P<0.001)$, the association was no longer significant after covariate adjustment for age, gender, years of education, body mass index, smoking, activities of daily living (ADLs), instrumental ADLs, physical activity, geriatric depression scale, and medical conditions. ${ }^{57}$

\section{DISCUSSION}

This review considered observational studies investigating the association between cognitive performance and hand motor function, with special consideration to those that evaluated handgrip strength and hand dexterity. The findings indicate a significant association between handgrip strength and cognitive performance or hand dexterity and cognitive performance; however, no studies evaluated the association among the three variables. Therefore, the present review has been outlined according to the findings for handgrip strength and hand dexterity separately.

Most of the longitudinal studies evaluated the association between either baseline handgrip strength and cognitive performance changes or vice-versa; however, only two studies ${ }^{52,53}$ analyzed both directions of the association. Because it is unclear which variable (handgrip strength or cognitive performance) influences the other in the long-term, it is necessary to consider the baseline effects of each variable on the changes of the other variable of interest. This also applies to the association between hand dexterity and cognitive performance, in that their association was examined only cross-sectionally. ${ }^{28,38-40}$ It would help to evaluate the influence of baseline hand dexterity and baseline cognitive function on subsequent changes in these domains.

Regarding cognitive performance, about $63.6 \%$ of all studies considered global cognition in their measurements, with the MMSE as the preferred test, whereas $36.4 \%$ considered other cognitive domains (Table 3). The studies that evaluated global cognition seemed to focus on the onset of cognitive impairment as a predictor of the association with handgrip strength. In this regard, a cross-sectional study using a subtest of the MMSE to define cognitive impairment ${ }^{31}$ reported no association between global cognition and handgrip strength, suggesting that cognitive evaluation should not be limited to global cognitive performance but should also include complex cognitive domains. By doing this, it would be possible to elucidate what cognitive domains relate to handgrip strength and hand dexterity. Furthermore, the cognitive test employed to detect cognitive impairment in community dwellers must be appropriately selected. Contrary to the studies included in this review, some studies suggest using the MMSE for screening dementia and the MoCA test for screening $\mathrm{MCI},{ }^{58}$ whereas the CASI test is claimed to be more sensitive than the MMSE for screening dementia in other studies. ${ }^{56}$

Concerning hand motor function, all of the studies included in this review (Table 1 and Table 2) measured handgrip strength as part of a physical function test battery to evaluate whole-body muscle strength or as a composite score to discriminate frailty. The studies acknowledge the association between handgrip strength and global cognitive performance, while the association with other cognitive domains needs to be observed further. On the other side, tests of hand dexterity, whose completion requires high cognitive demand as well as complex sensorimotor coordination, such as eye-finger coordination, ${ }^{28,30,59}$ were evaluated not only in community-dwelling older adults but also in neuromuscular disease patients to evaluate fine motor coordination skills. ${ }^{38,40}$ Attention seems to be another key factor associated with hand dexterity performance, ${ }^{38,39}$ which may be of interest for further evaluation in community-dwelling older adults. Therefore, it may be implied that hand dexterity test scores reflect complex functional aspects of motor and cognitive demands, such as attention, speed, and coordination. This assumption is in accordance with a previous study ${ }^{59}$ suggesting that hand function should consider not only the assessment of grip strength but also the upper motor coordination and sensorimotor processing domains.

Another point to consider is the covariate adjustment. In this regard, Veronese et $\mathrm{al}^{57}$ found no significant association between handgrip strength and cognitive impairment after adjusting for 
Kobayashi-Cuya KE, et al.

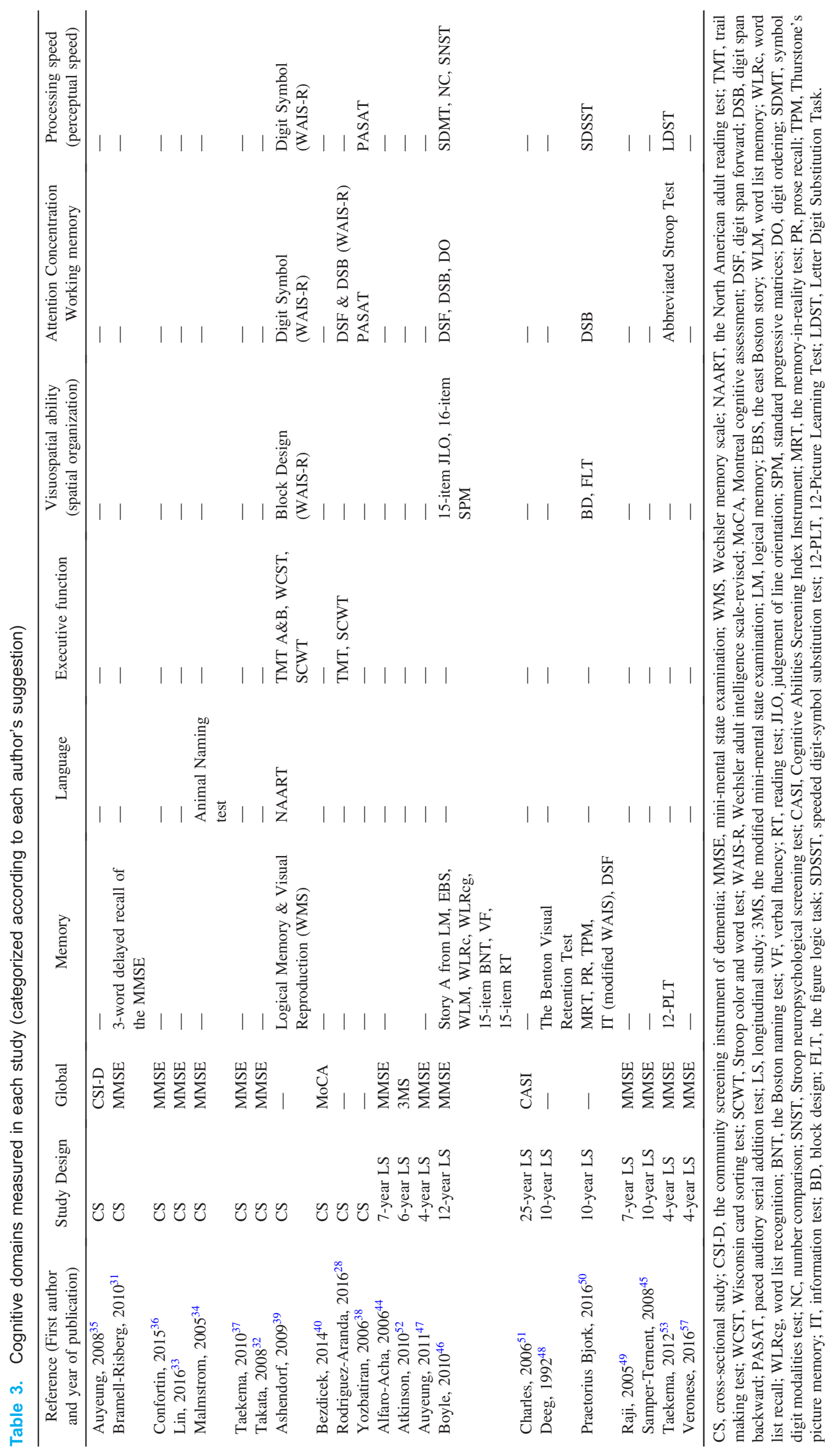


covariates. It is important to note that, unlike the other longitudinal studies included in this review, this study considered health behavior covariates, such as smoking, drinking, and exercise, besides demographic covariates and medical conditions. This assumption is supported by Atkinson et $\mathrm{al}^{52}$ who found no association between baseline handgrip strength and cognitive performance changes, after statistical adjustment for health behavior covariates. This is, however, not supported by crosssectional studies in that these variables did not affect the significance of the relationship. Unlike cross-sectional studies, longitudinal studies are better at analyzing certain pattern of behaviors and may suggest other factors that may influence the association of interest. Therefore, we suggest caution when using covariates, especially in long-term studies, in that they may change the interpretation of the results.

\section{Strengths and limitations of the review}

The strengths of this systematic review include the examination of the integrated assessment of hand motor function (handgrip strength and hand dexterity) and its relation to cognitive function in community-dwelling older adults. This review also provides an understanding of the current methodological approaches used for examining the association in question. However, the findings of the current review need to be interpreted with caution. First, we only examined studies that considered handgrip strength and hand dexterity as variables of hand function. Second, we have only searched articles written in English. Third, we did not perform a risk of bias assessment of the studies included in this review. Despite of these limitations, this review contributes to our understanding of the complexity of hand function as a sensorimotor and cognitive integrated mechanism and acknowledges hand motor function as a measurable indicator for the early detection of cognitive impairment in healthy older adults.

\section{Implications for future study}

Unlike handgrip strength, only cross-sectional studies evaluated the association between hand dexterity and cognitive performance. We suggest analyzing this association in the long-term to elucidate the ways in which hand dysfunction may be related to cognitive impairment. Also, most of the cross-sectional studies evaluating hand dexterity included small sample sizes (less than $50)$; therefore, it is necessary to increase the sample size in community dwellers to make it more representative. We also recommend grouping the samples into narrower age groups (ie, 70-74, 75-79 years old), especially if they come from a small sample size, considering that cognitive performance may be more vulnerable to changes as we age. ${ }^{60}$ Finally, by analyzing the cross-sectional and longitudinal associations between hand dexterity, handgrip strength, and cognitive domains in older adults, there would be a better understanding of the mechanisms related to cognitive decline in late adulthood.

\section{Conclusions}

Handgrip strength associates with cognitive performance crosssectionally and longitudinally; however, it is unclear to speculate which variable-having weak handgrip strength or having low cognitive performance at baseline - affects the other in the longterm. Hand dexterity associates with cognitive performance crosssectionally in community-dwelling older adults. Most of the studies utilized global cognition as an indicator of cognitive performance; therefore, long-term studies measuring different cognitive domains, as well as hand dexterity tests other than pegboard tests, such as line tracing and hand steadiness, are needed.

\section{ACKNOWLEDGEMENTS}

Conflicts of interest: None declared.

\section{REFERENCES}

1. Greiner PA, Snowdon DA, Schmitt FA. The loss of independence in activities of daily living: the role of low normal cognitive function in elderly nuns. Am J Public Health. 1996;86:62-66.

2. Talmelli LF, Gratão AC, Kusumota L, Rodrigues RA. Functional independence level and cognitive deficit in elderly individuals with Alzheimer's disease. Rev Esc Enferm USP. 2010;44:933-939.

3. Farias ST, Mungas D, Reed BR, Harvey D, DeCarli C. Progression of mild cognitive impairment to dementia in clinic- vs communitybased cohorts. Arch Neurol. 2009;66:1151-1157.

4. Roberts RO, Knopman DS, Mielke MM, et al. Higher risk of progression to dementia in mild cognitive impairment cases who revert to normal. Neurology. 2014;82:317-325.

5. WHO. WHO | Dementia: a public health priority. WHO. 2016

6. Peracino A, Pecorelli S. The epidemiology of cognitive impairment in the aging population: implications for hearing loss. Audiol Neurootol. 2016;21(Suppl 1):3-9.

7. Roy S, Ficarro S, Duberstein P, et al. Executive function and personality predict instrumental activities of daily living in Alzheimer disease. Am J Geriatr Psychiatry. 2016;24:1074-1083.

8. Zucchella C, Bartolo M, Bernini S, Picascia M, Malinverni P, Sinforiani E. Modeling Alzheimer's disease through functional independence and participation. Alzheimer Dis Assoc Disord. 2017;31(3):218-224.

9. Lee J, Lee B, Park Y, Kim Y. Effects of combined fine motor skill and cognitive therapy to cognition, degree of dementia, depression, and activities of daily living in the elderly with Alzheimer's disease. J Phys Ther Sci. 2015;27:3151-3154.

10. Aggarwal NT, Wilson RS, Beck TL, Bienias JL, Bennett DA. Motor dysfunction in mild cognitive impairment and the risk of incident Alzheimer disease. Arch Neurol. 2006;63:1763-1769.

11. Aihie Sayer A, Osmond C, Briggs R, Cooper C. Do all systems age together? Gerontology. 1999;45:83-86.

12. Deary IJ, Corley J, Gow AJ, et al. Age-associated cognitive decline. Br Med Bull. 2009;92:135-152.

13. Kelley BJ, Petersen RC. Alzheimer's disease and mild cognitive impairment. Neurol Clin. 2007;25:577-609, v.

14. Petersen RC, Doody R, Kurz A, et al. Current concepts in mild cognitive impairment. Arch Neurol. 2001;58:1985-1992.

15. Metzger FG, Hobert MA, Ehlis AC, et al; TREND Study team. Dual tasking for the differentiation between depression and mild cognitive impairment. Front Aging Neurosci. 2016;8:235.

16. Sakurai R, Fujiwara Y, Yasunaga M, et al. Regional cerebral glucose metabolism and gait speed in healthy community-dwelling older women. J Gerontol A Biol Sci Med Sci. 2014;69:1519-1527.

17. Cottone C, Porcaro C, Cancelli A, Olejarczyk E, Salustri C, Tecchio F. Neuronal electrical ongoing activity as a signature of cortical areas. Brain Struct Funct. 2017;222(5):2115-2126.

18. Dettmers C, Fink GR, Lemon RN, et al. Relation between cerebral activity and force in the motor areas of the human brain. $J$ Neurophysiol. 1995;74:802-815.

19. Hupfeld KE, Ketcham CJ, Schneider HD. Transcranial direct current stimulation (tDCS) to the supplementary motor area (SMA) influences performance on motor tasks. Exp Brain Res. 2017;235(3): 851-859.

20. Jaeger L, Marchal-Crespo L, Wolf P, Riener R, Michels L, Kollias S Brain activation associated with active and passive lower limb stepping. Front Hum Neurosci. 2014;8:828.

21. Metter EJ, Conwit R, Metter B, Pacheco T, Tobin J. The relationship 
of peripheral motor nerve conduction velocity to age-associated loss of grip strength. Aging (Milano). 1998;10:471-478.

22. Quandt F, Bönstrup M, Schulz R, et al. Spectral variability in the aged brain during fine motor control. Front Aging Neurosci. 2016;8:305

23. Haggard P. Conscious intention and motor cognition. Trends Cogn Sci. 2005;9:290-295.

24. Leisman G, Moustafa AA, Shafir T. Thinking, walking, talking: integratory motor and cognitive brain function. Front Public Health. 2016;4:94

25. Kluger A, Gianutsos JG, Golomb J, et al. Patterns of motor impairement in normal aging, mild cognitive decline, and early Alzheimer's disease. J Gerontol B Psychol Sci Soc Sci. 1997; 52B(1):P28-P39.

26. de Paula JJ, Albuquerque MR, Lage GM, Bicalho MA, RomanoSilva MA, Malloy-Diniz LF. Impairment of fine motor dexterity in mild cognitive impairment and Alzheimer's disease dementia: association with activities of daily living. Rev Bras Psiquiatr. 2016;38:235-238

27. Cole KJ, Beck CL. The stability of precision grip force in older adults. J Mot Behav. 1994;26:171-177.

28. Rodríguez-Aranda C, Mittner M, Vasylenko O. Association between executive functions, working memory, and manual dexterity in young and healthy older adults: an exploratory study. Percept Mot Skills. 2016;122:165-192.

29. Van Halewyck F, Lavrysen A, Levin O, Boisgontier MP, Elliott D, Helsen WF. Factors underlying age-related changes in discrete aiming. Exp Brain Res. 2015;233:1733-1744.

30. Martin JA, Ramsay J, Hughes C, Peters DM, Edwards MG. Age and grip strength predict hand dexterity in adults. PLoS One. 2015;10: e0117598

31. Bramell-Risberg E, Jarnlo GB, Elmståhl S. Slowing of alternating forearm movements is associated with cognitive impairment in community-dwelling older people. Dement Geriatr Cogn Disord. 2010;29:457-466.

32. Takata Y, Ansai T, Soh I, et al. Physical fitness and cognitive function in an 85-year-old community-dwelling population. Gerontology. 2008;54:354-360.

33. Lin PS, Hsieh CC, Cheng HS, Tseng TJ, Su SC. Association between physical fitness and successful aging in Taiwanese older adults. PLoS One. 2016;11:e0150389.

34. Malmstrom TK, Wolinsky FD, Andresen EM, Miller JP, Miller DK. Cognitive ability and physical performance in middle-aged African Americans. J Am Geriatr Soc. 2005;53:997-1001.

35. Auyeung TW, Kwok T, Lee J, Leung PC, Leung J, Woo J. Functional decline in cognitive impairment-the relationship between physical and cognitive function. Neuroepidemiology. 2008:31:167-173

36. Confortin SC, Barbosa AR. Factors associated with muscle strength among rural community-dwelling older women in Southern Brazil. J Geriatr Phys Ther. 2015;38:162-168.

37. Taekema DG, Gussekloo J, Maier AB, Westendorp RG, de Craen AJ. Handgrip strength as a predictor of functional, psychological and social health. A prospective population-based study among the oldest old. Age Ageing. 2010;39:331-337.

38. Yozbatiran N, Baskurt F, Baskurt Z, Ozakbas S, Idiman E. Motor assessment of upper extremity function and its relation with fatigue, cognitive function and quality of life in multiple sclerosis patients. J Neurol Sci. 2006;246:117-122.

39. Ashendorf L, Vanderslice-Barr JL, McCaffrey RJ. Motor tests and cognition in healthy older adults. Appl Neuropsychol. 2009;16:171176.

40. Bezdicek O, Nikolai T, Hoskovcová M, et al. Grooved pegboard predicates more of cognitive than motor involvement in Parkinson's disease. Assessment. 2014;21:723-730.

41. Earhart GM, Cavanaugh JT, Ellis T, Ford MP, Foreman KB, Dibble L. The 9-hole PEG test of upper extremity function: average values, test-retest reliability, and factors contributing to performance in people with Parkinson disease. J Neurol Phys Ther. 2011;35:157163.

42. Tombaugh TN. A comprehensive review of the Paced Auditory Serial Addition Test (PASAT). Arch Clin Neuropsychol. 2006;21: 53-76.

43. Gronwall DM. Paced auditory serial-addition task: a measure of recovery from concussion. Percept Mot Skills. 1977;44:367-373.

44. Alfaro-Acha A, Al Snih S, Raji MA, Kuo YF, Markides KS, Ottenbacher KJ. Handgrip strength and cognitive decline in older Mexican Americans. J Gerontol A Biol Sci Med Sci. 2006;61:859865 .

45. Samper-Ternent R, Al Snih S, Raji MA, Markides KS, Ottenbacher KJ. Relationship between frailty and cognitive decline in older Mexican Americans. J Am Geriatr Soc. 2008;56:1845-1852.

46. Boyle PA, Buchman AS, Wilson RS, Leurgans SE, Bennett DA. Physical frailty is associated with incident mild cognitive impairment in community-based older persons. J Am Geriatr Soc. 2010;58:248-255.

47. Auyeung TW, Lee JS, Kwok T, Woo J. Physical frailty predicts future cognitive decline-a four-year prospective study in 2737 cognitively normal older adults. J Nutr Health Aging. 2011;15:690694.

48. Deeg DJ, Haga H, Yasumura S, Suzuki T, Shichita K, Shibata H. Predictors of 10-year change in physical, cognitive and social function in Japanese elderly. Arch Gerontol Geriatr. 1992;15:163179.

49. Raji MA, Kuo YF, Snih SA, Markides KS, Peek MK, Ottenbacher KJ. Cognitive status, muscle strength, and subsequent disability in older Mexican Americans. J Am Geriatr Soc. 2005;53:1462-1468.

50. Praetorius Björk M, Johansson B, Hassing LB. I forgot when I lost my grip-strong associations between cognition and grip strength in level of performance and change across time in relation to impending death. Neurobiol Aging. 2016;38:68-72.

51. Charles LE, Burchfiel CM, Fekedulegn D, et al. Occupational and other risk factors for hand-grip strength: the Honolulu-Asia Aging Study. Occup Environ Med. 2006;63:820-827.

52. Atkinson HH, Rapp SR, Williamson JD, et al. The relationship between cognitive function and physical performance in older women: results from the women's health initiative memory study. J Gerontol A Biol Sci Med Sci. 2010;65:300-306.

53. Taekema DG, Ling CH, Kurrle SE, et al. Temporal relationship between handgrip strength and cognitive performance in oldest old people. Age Ageing. 2012;41:506-512.

54. Benton AL. The visual retention test as a constructional praxis task. Confin Neurol. 1962;22:141-155.

55. Woods DL, Kishiyamaa MM, Lund EW, et al. Improving digit span assessment of short-term verbal memory. J Clin Exp Neuropsychol. 2011:33:101-111

56. Teng EL, Hasegawa K, Homma A, et al. The Cognitive Abilities Screening Instrument (CASI): a practical test for cross-cultural epidemiological studies of dementia. Int Psychogeriatr. 1994;6:4558; discussion 62.

57. Veronese N, Stubbs B, Trevisan C, et al. What physical performance measures predict incident cognitive decline among intact older adults? A 4.4 year follow up study. Exp Gerontol. 2016;81:110-118.

58. Tsai JC, Chen $\mathrm{CW}$, Chu $\mathrm{H}$, et al. Comparing the sensitivity, specificity, and predictive values of the Montreal Cognitive Assessment and Mini-Mental State Examination when screening people for mild cognitive impairment and dementia in Chinese population. Arch Psychiatr Nurs. 2016;30:486-491.

59. Lawrence EL, Dayanidhi S, Fassola I, et al. Outcome measures for hand function naturally reveal three latent domains in older adults: strength, coordinated upper extremity function, and sensorimotor processing. Front Aging Neurosci. 2015;7:108.

60. Gunstad J, Paul RH, Brickman AM, et al. Patterns of cognitive performance in middle-aged and older adults: a cluster analytic examination. J Geriatr Psychiatry Neurol. 2006;19:59-64. 